\title{
Dinamismo de capacidades mediante el fomento de estudios de posgrado para la docencia universitaria
}

\section{Dynamism of capacities through the promotion of postgraduate studies for university teaching}

\author{
MSc, Fabián Alberto Gordillo Manssur ${ }^{1}$ \\ fabian.gordillom@ug.edu.ec \\ MSc, Ivette Cristina Gordillo Manssur ${ }^{2}$ \\ ivettecgm@hotmail.com \\ MSc, Ana María Campuzano Vera \\ anmacamp@espol.edu.ec
}

Recibido: 1/04/2018, Aceptado: 1/06/2018

\begin{abstract}
RESUMEN
El posgrado es un elemento para el desarrollo en la calidad universitaria. La calidad universitaria es: docencia, investigación y extensión, en donde el docente es el responsable. El objetivo fue evaluar la importancia del nivel de enseñanza para el fortalecimiento y generación de las competencias y capacidades profesionales para el ejercicio de la docencia universitaria. La investigación que se realizó fue de tipo no experimental de manera cualitativa y cuantitativa con observación con visita in situ y modelo de encuesta para valorar su vinculación con la investigación, valoración de aprendizaje y perfiles de ingreso de estudiante mediante criterios de evaluación. Los estudios de posgrado generan competencias y capacidades, las cuáles no necesariamente son aplicadas en la labor docente; sin embargo, existe un compromiso por el mejoramiento de la calidad de la educación, e indican los docentes que ni el salario, respeto y continuidad son cualidades que los motiven o adquieran.
\end{abstract}

Palabras clave: Competencias laborales, docencia, universidad

\footnotetext{
1 Universidad de Guayaquil, Ecuador

2 Universidad de Guayaquil, Ecuador

3 Escuela Superior Politécnica del Litoral, Ecuador
} 


\begin{abstract}
The postgraduate course is an element for the development of university quality. The university quality is: teaching, research and extension, where the teacher is responsible. The objective was to evaluate the importance of the level of education for the strengthening and generation of professional skills and abilities for the exercise of university teaching. The research that was carried out was of a nonexperimental type in a qualitative and quantitative way with observation with an onsite visit and a survey model to assess its link with research, assessment of learning and student income profiles through evaluation criteria. Postgraduate studies generate competences and abilities, which are not necessarily applied in the teaching work; However, there is a commitment to improve the quality of education, and teachers indicate that neither salary, respect and continuity are qualities that motivate or acquire them.
\end{abstract}

Keywords: Labor competences, teaching, university

\title{
Introducción
}

En América Latina, el posgrado comenzó siendo una actividad anexa y no sólidamente enlazada a la formación profesional, sin embargo la educación de posgrado, constituye el conjunto de procesos de enseñanza-aprendizaje dirigidos a garantizar la preparación de los graduados universitarios, con el propósito de completar, actualizar y profundizar en los conocimientos y habilidades que poseen, y alcanzar un mayor nivel de ejercicio profesional o de conocimiento y habilidades científicas, en correspondencia con los avances científico-técnicos y las necesidades de las entidades en que laboran. En la actualidad, es considerado un elemento fundamental para lograr el desarrollo pertinente, ya que es percibida como una inversión rentable e instrumento estratégico importante en las políticas de desarrollo acelerado principalmente ligado a la calidad universitaria.

El concepto de calidad universitaria resume tres dimensiones diferentes: docencia, investigación y extensión, que contempla la valoración del personal docente, de sus programas, de sus métodos de enseñanza-aprendizaje, sus aspectos metodológicos, de la investigación para el desarrollo y como indicador de cambio y de mejora de la organización y administración del ambiente académico, de los estudiantes, del contexto institucional y del impacto en la sociedad (Manzo, Rivera y Rodríguez, 2006).

Las exigencias actuales relacionan la profesión docente con la mejora de la calidad. Puede ser que la función docente sea de calidad para determinados usuarios y para otros no, que se centre en los objetivos, en los resultados, en los recursos, en las demandas sociales. Podemos hablar de calidad en la profesión docente, en los aprendizajes, en la infraestructura del sistema, en los procesos educativos, en los objetivos y resultados académicos (Duque, García y Labao, 2012).

\section{Desarrollo}

Cuando hablamos del docente universitario estamos hablando del responsable de la enseñanza, innovación, investigación y tecnología en las clases dentro de una 


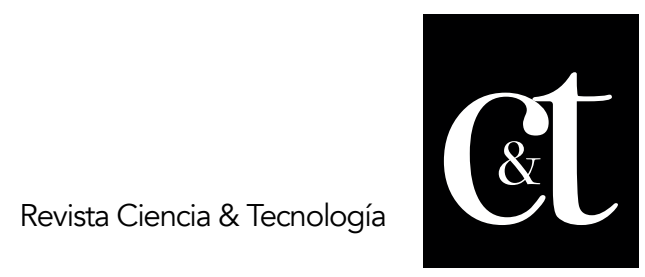

No. 19, 31 de julio de 2018

ISSN impreso: 1390 - 6321

carrera universitaria; es quien conduce la clase acorde a una metodología de enseñanza. Los docentes universitarios, en su mayoría se sitúan en el tipo de profesor tecnológico, su objetivo fundamental es la transmisión de conocimientos, cercado por la planificación cerrada e inflexible, que busca solo el cumplimiento de un programa educativo sin importan la construcción del conocimiento en los estudiantes y su formación como entes reflexivos, críticos y constructivos de su propio conocimiento, lo cual ya no es aplicable (Silverio, 2012 y Leyva et al, 2015).

La atención sobre la calidad de la docencia y los procesos pedagógicos en las instituciones de educación superior (IES) aglutinan esfuerzos en la renovación de las metodologías educativas. Se deseaba, así, la sustitución del modelo centrado en la enseñanza por otro centrado en el aprendizaje (Villarroel y Bruna, 2014). Si bien es cierto que muchas de las IES cumplen con los requisitos mínimos de formación del personal académico, y de que el reclutamiento y selección de este se hace por lo general con base en los reglamentos establecidos, no es menos cierto que la calidad de una gran parte de los docentes no es la más idónea en términos de experiencia y de formación pedagógica y especializada en las áreas en que imparten docencia (pertinencia) (Silie, Cuello y Mejía, 2003).

La necesidad en la pertinencia en los procesos de enseñanza y aprendizaje, hacen que el estudio de posgrado se conciba potencialmente como la preparación metodológica para la investigación, el desarrollo de esta y su vinculación con aquellos sectores de la sociedad que requieren de nuevos conocimientos, desarrollos tecnológicos, científicos y/o innovaciones (Reynaga, 2012: 25). Así, la finalidad de los estudios de posgrado es contribuir a la formación de un profesional en una disciplina o área de conocimiento para que desarrolle la capacidad de emprender proyectos de investigación, así como para aplicar e innovar el conocimiento científico aunado a la resolución de problemas en su campo de acción con la intención de actualizar y profundizar los conocimientos y habilidades que poseen (Hernández, Tavera y Jiménez, 2012; Jaramillo, 2009).

Por tanto, los posgrados poseen una función endógena y exógena. La primera se relaciona con la preparación de personal calificado que posibilite que este nivel educativo permanezca y se desarrolle a través de la docencia y de la creación e implementación de proyectos de investigación. En tanto que la segunda, se refiere a la aplicación de la generación de los conocimientos científicos y tecnológicos en el sector productivo, social y de servicios (Jaramillo, 2009).

Es importante para evaluar las competencias de un docente de posgrado es preciso hacerlo de una forma holística considerando en forma interrelacionada las funciones de docencia, investigación, planeación y evaluación como tareas interconectadas (Mas, 2011 y Tejada, 2009). Así, el docente de este nivel no queda circunscrito a un solo ámbito de actuación por lo que es relevante identificar como dichas competencias se han implementado en los programas de posgrado a fin de establecer su relevancia e impacto no sólo en la formación de recursos humanos sino también en la calidad educativa de las IES (Cardoso y Cerecedo, 2011). 


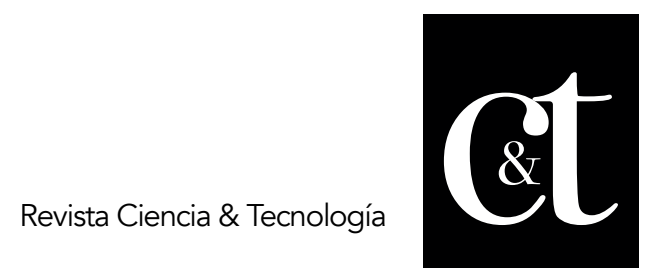

No. 19, 31 de julio de 2018

ISSN impreso: 1390 - 6321

La transferencia de la tecnología e ideología extranjeras, por lo general, se considera un factor esencial del desarrollo en los estudios avanzados, no obstante, este camino exige tener en cuenta las características y necesidades profesionales en el contexto latinoamericano, por lo cual es necesario concientizar a políticos y gobernantes, autoridades universitarias y al sector productivo, acerca de la importancia y necesidad social de estructurar políticas y sistemas de estudios de posgrado que permitan la inserción pertinente de esta actividad en los procesos de dominio y creación del saber científico, técnico y humanístico. El objetivo del presente estudio se encaminó a evaluar la importancia del nivel de enseñanza posgrado como requerimiento fundamental para el fortalecimiento y generación de las competencias y capacidades profesionales indispensables para el ejercicio de la docencia universitaria.

\section{Método}

El profesor, estudiante de posgrado para realizar una práctica docente de calidad, es necesario que efectúe la función de planeación, en la medida que tiene que diseñar, y ajustar sus asignaturas con base en los perfiles de ingreso de sus alumnos; así mismo, así como la de evaluación es preponderante porque requiere valorar los aprendizajes y logros adquiridos por el grupo. Por último, la función de investigación porque es relevante el diseño e implementación de proyectos de innovación que posibiliten el avance científico de las diversas áreas del conocimiento en relación con el sector productivo y de servicios (Jaramillo, 2009).

De acuerdo con esta premisa, se evaluaron todos los planes de clase y de práctica de la carrera de ingeniería agronómica de la Facultad de Ciencias Agrarias, con la finalidad de valorar su vinculación con la investigación, valoración de aprendizaje y perfiles de ingreso de estudiante. Por lo expuesto, se establecieron criterios de evaluación, como, por ejemplo: resultados de aprendizaje, competencias adquiridas y relación de la práctica de investigación con los contenidos de la asignatura.

Para la evaluación se contó con seis tipos de evaluadores: la participación del docente encargado de la asignatura, un evaluador externo con competencias de la asignatura, docente de la asignatura prerrequisito y docente de la asignatura posterior de acuerdo al flujograma de la carrera, coordinador de investigación de la facultad y una autoridad de la carrera o facultad (coordinador de carrera, vicedecano o decano).

La investigación que se realizó fue de tipo no experimental de manera cualitativa y cuantitativa con observación con visita in situ y modelo de encuesta, porque se enfocó en el diagnóstico de las competencias docentes en el nivel de posgrado, específicamente en el campo de la agricultura. Asimismo, fue no experimental porque se centró, en observar las dimensiones que componen a las competencias y capacidades de los docentes en estudio de posgrado. Además, se realizó una evaluación de las cualidades que los docentes adquieren al ingresar a un programa de posgrado de acuerdo al nivel y el área del posgrado en una muestra representativa de 100 docentes. 


\section{Resultados}

Los resultados indican que el $60 \%$ de los planes no se encontraban con prácticas con el componente de investigación, el $70 \%$ no contenían dentro de los resultados esperados los resultados del componente de investigación, $20 \%$ no representaban las competencias adquiridas por los docentes.

De acuerdo a los resultados de aprendizaje, se obtuvo que el $90 \%$ de las asignaturas presentaron trabajos de investigación en congresos, seminarios e intercambio, representando las competencias adquiridas durante el semestre de acuerdo a las capacidades y competencias trasmitidas por los docentes de acuerdo a su ámbito de estudio de posgrado. Además, este nuevo enfoque les permite a los estudiantes adquirir y afianzar conocimientos investigativos cada semestre y culminar el aprendizaje en su trabajo de fin de grado.

Luego, mediante el análisis posterior de las cualidades que los docentes adquieren con estudios de posgrado, encontramos que las principales cualidades son: diferenciación, nuevos conocimientos, cambios de perspectiva, contactos, acceso al mercado laboral y experiencia de acuerdo a nivel de posgrado doctorado y en el área técnica (tabla 1).

Tabla 1. Cualidades adquiridas por docentes y la influencia del posgrado

\begin{tabular}{lccc}
\hline \multicolumn{1}{c}{ Cualidad } & Docentes & $\begin{array}{c}\text { Nivel de } \\
\text { posgrado }\end{array}$ & Área de estudio \\
\hline Diferenciación & 93 & Doctorado & Básica \\
Nuevos conocimientos & 90 & Doctorado & Técnica \\
Cambios de perspectiva & 100 & $\begin{array}{c}\text { Doctorado } \\
\text { Doctorado }\end{array}$ & $\begin{array}{c}\text { Administrativa } \\
\text { Técnica }\end{array}$ \\
Contactos & 90 & Doctorado & \\
Acceso al mercado & 100 & Doctorado & Técnica \\
laboral & & Maestria & Administrativa \\
Experiencia & 96 & Maestria & Básica \\
Salario & 85 & Maestria & Básica \\
Respeto & 80 & & \\
Continuidad & 80 & & \\
\hline
\end{tabular}

Fuente: elaboración propia

\section{Conclusiones}

Los estudios de posgrado generan competencias y capacidades en los docentes, las cuáles no necesariamente son aplicadas en la labor docente; sin embargo, existe un compromiso por el mejoramiento de la calidad de la educación por medio del desarrollo de una nueva planificación de acuerdo a la pertinencia y el perfil de ingreso de los estudiantes; $y$, diseño y desarrollo de proyectos de investigación, lo cual concuerda con lo establecido por Cardoso, Cerecedo y Vanegas, (2013) quienes indican que en un estudio realizado para evaluar las competencias de docentes en programa de posgrado en administración, el $81.4 \%$ está totalmente de acuerdo con diseñar y desarrollar proyectos de investigación institucionales para el avance científico; y Cuervo, Noriega y Martínez (2012) en un estudio para diagnosticar 


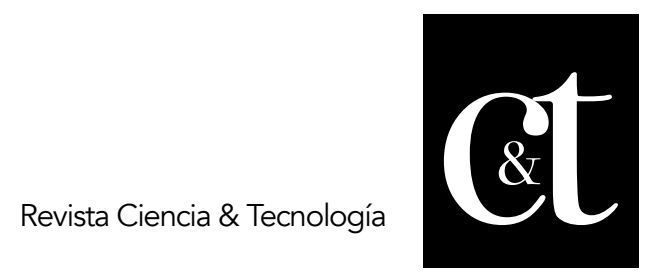

No. 19, 31 de julio de 2018

ISSN impreso: 1390 - 6321

competencias científicas en estudiantes de posgrado de ciencias naturales e ingenierías, los resultados señalan que desde la perspectiva de los estudiantes, los posgrados cumplen su función de formadores de científicos, aunque deben realizar acciones para mejorar la competencia relacionada con la gestión de recursos y, en general, para procurar que la importancia que los estudiantes brindan a las competencias en el currículo sea acorde con el desarrollo alcanzado por ellos.

De acuerdo a las cualidades adquiridas por la mayoría de los docentes cuyo nivel es o están en un doctorado y cuya área de estudio es técnica indican que ni el salario, respeto y continuidad son cualidades que los motiven o adquieran con el doctorado; ya que, el salario aumenta de acuerdo a la normativa actual, el respeto siempre debe y existe entre los docentes, y normalmente los docentes que se encuentran en estudios doctorales son docentes con nombramiento en las instituciones por ende la continuidad laboral no es algo que se asegure, caso contrario con los docentes inmersos en estudios de posgrado nivel maestría y otras áreas de estudio. Las diferencias entre los tres tipos de posgrado se vinculan fundamentalmente con las características y nivel de profundización de los conocimientos; las maestrías tienen por objeto proporcionar una formación académica y/o profesional, profundizando el conocimiento teórico, metodológico, tecnológico, de gestión o artístico en el ámbito de una disciplina $o$ área interdisciplinaria 0 de un campo profesional o multiprofesional, en cambio, el doctorado requiere la realización de un trabajo de tesis que signifique una contribución original al conocimiento de la especialización científica y/o tecnológica elegida, esta diferenciación que se menciona es percibida por las exigencias de la educación actual.

\section{Referencias bibliográficas}

Cardoso, E., Cerecedo, M., y Vanegas, E. (2013). Las competencias docentes en los programas de posgrado en Administración: Un Estudio de Diagnóstico. Formación universitaria, 6(2), 43-50.

Cardoso, E., y Cerecedo, M. (2011). Propuesta de indicadores para evaluar la calidad de un programa de posgrado en educación. Revista Electrónica de Investigación Educativa, 13(2), 68 - 82, 2011, http://redie.uabc.mx/vol13no2/contenido-cardosocerecedo.html.

Cuervo, A., Noriega, Á., y Martínez, A. (2012). Competencias científicas en estudiantes de posgrado de Ciencias Naturales e Ingenierías. Sinéctica, (39).

Duque, P., García, P., y Labao, T. (2012). Nuevos retos de la profesión docente. En Silvero, M. Retos del docente universitario del siglo XXI.

Hernández, C., Tavera, M. y Jiménez, M. (2012). Seguimiento de Egresados en Tres Programas de Maestría en una Escuela del Instituto Politécnico Nacional en México. Revista Formación Universitaria, 5(2), 41 - 52, 2012, https://scielo.conicyt.cl/pdf/formuniv/v5n2/art06.pdf.

Jaramillo, H. (2009). La formación de posgrado en Colombia: maestrías y doctorados. Revista Ciencia, Tecnología y Sociedad, 13(5), 131-155.

Gordillo, Gordillo, Campuzano. Dinamismo de capacidades mediante el fomento de estudios de posgrado para la docencia universitaria 
Leyva, O., Ganga, F., Tejada, J., Paz, H., y Alfredo, A. (2015). La formación por competencias en la educación superior: alcances y limitaciones desde referentes de México, España y Chile.

Manzo, L., Rivera, C., y Rodríguez, A. (2006). La educación de posgrado y su repercusión en la formación del profesional iberoamericano. Educación Médica Superior, 20(3).

Mas, O. (2011). El profesor universitario: sus competencias y formación. Profesorado. Revista de Currículum y Formación de Profesorado, 15(3), 1 - 17.

Reynaga, S. (2012). El posgrado en México: Tensiones entre la diversificación y la dispersión. En M. Serna y R. Pérez (Eds.) Logros e innovación en el posgrado. México: COMEPO, 25 - 31.

Silie, R., Cuello, C., y Mejía, M. (2003). Estudio sobre la calidad de la educación superior en la República Dominicana. Santo Domingo: SEESCYT/IESALC.

Tejada, J. (2009). El trabajo por competencias en el prácticum: cómo organizarlo y cómo evaluarlo. Revista Electrónica de Investigación Educativa, 7 (2), 56 - 70. http://redie.uabc.mx/vol7no2/contenido-tejada.html.

Villarroel, V., y Bruna, D. (2014). Reflexiones en torno a las competencias genéricas en educación superior: Un desafío pendiente. Psicoperspectivas, $13(1), 22-34$. 